the solution of a certain partial differential equation of the second order which is reduced by quadratures to a linear partial differential equation of the first order.

The more complicated types of problems in the calculus of variations are examined in a similar manner. But in no type except the simplest are the differential equations which present themselves as necessary con ditions of general character, $i$. e., the system of extremals is no longer an arbitrary system of curves or surfaces (in space of any number of dimensions). Thus an integral of type

$$
\int F\left(x, y, y^{\prime}, y^{\prime \prime}\right) d x
$$

leads to a four parameter system of extremals; but only a restricted class of four parameter systems of curves can be obtained in this way. The characterization of this class depends upon the consistency of a certain set of partial differential equations.

The results obtained are of interest in connection with Hilbert's recent investigations, as serving to characterize the types of differential equations which can be studied by means of corresponding problems in the calculus of variations. All ordinary equations of the second order, but only a restricted class of linear partial differential equations of the second order, can be so treated.

Columbia University.

Edward Kasner, Assistant Secretary.

\title{
MODERN METHODS OF TREATING DYNAMICAL PROBLEMS AND IN PARTICULAR THE PROBLEM OF THREE BODIES.
}

\author{
PRÉCIS OF FOUR LECTURES DELIVERED BEFORE THE \\ ITHACA COLLOQUIUM, AUGUST 21-24, 1901.
}

BY PROFESSOR E. W. BROWN.

IN this course an attempt was made to give some idea of the methods which have been used during the last twentyfive years to obtain information about certain classes of dynamical problems and in particular about those problems which are ordinarily considered by physicists to be insoluble in all their generality. One of the most important of these problems and one specially considered in the lectures is the Problem of Three Bodies, which at 
present can only be attacked in special cases. Attention was mainly directed to the methods-initiated by G. W. Hill, H. Gyldén, H. Poincaré, and others-and in order that these might be set forth, proofs were generally omitted, except when they were necessary for purposes of illustration; thus the lectures became descriptive to a large extent. Two of the objects aimed at were to show how the methods of modern analysis have been applied with some success to dynamical problems and to indicate the general lines on which research has been and can be carried on. It was desired to make the course interesting to an audience mainly composed of pure mathematicians. As no special knowledge on the part of the audience was assumed, it was necessary to devote most of the first lecture to the general principles of dynamics. The other topics selected were the existence of integrals, invariant integrals, divergent series, periodic solutions in general and those of the first class, characteristic exponents, periodic solutions of the second class and of the second species, asymptotic solutions and stability. There were four sessions, each of nearly two hours, with a ten minute interval in the middle.

1. General Principles of Dynamics.*-The laws of motion and the energy equation were briefly touched on. The equations of dynamics can be put into a canonical form

$$
\frac{d x_{i}}{d t}=\frac{\partial F}{\partial y_{i}}, \quad \frac{d y_{i}}{d t}=-\frac{\partial F}{\partial x_{i}} \quad(i=1,2, \cdots, n),
$$

where $x_{i}, y_{i}$ are coördinates defining the position and motion of the system and $F$ is a function of them. The transformations (tangential) which retain the canonical form when the defining coördinates are altered, can be made by means of a certain function $S$; this function has important properties when the Jacobian method is used to solve the equations by continued approximation, the approximations being carried out by varying the arbitrary constants. The types of transformations most frequently used are the linear and the polar, the latter being of the form

$$
x^{\prime}=\sqrt{2 x} \cos y, \quad y^{\prime}=\sqrt{2 x} \sin y .
$$

In general, we can expand $F$ in a convergent series

\footnotetext{
* See the astronomical treatises of Tisserand, Dziobek, Poincaré, Brown and many works on theoretical mechanics.
} 


$$
F=F_{0}+\mu F_{1}+\mu^{2} F_{2}+\cdots \equiv F_{0}+R,
$$

where $\mu$ is a given constant. The treatment consists in obtaining a general solution with $F=F_{0}$ and then in considering the arbitrary constants as variable when $F=F_{0}+R$; in the Jacobian method the variable arbitraries satisfy a set of canonical equations. By first omitting $\mu^{2}, \mu^{3}, \cdots$ and then following out the same process, we can step by step approximate formally to the required solution. The application to astronomical problems consists in supposing the body under consideration to describe an ellipse in the first approximation $\left(F=F_{0}\right)$; the arbitraries are then functions of the elements of this ellipse. Delaunay's lunar theory and the usual method of treating the planetary theory are examples.

Poincaré has frequently used the Jacobian method in a different way.* The solution, in fact, depends on the discovery of a solution of the partial differential equation

$$
F\left(\frac{\partial S}{\partial y_{i}}, y_{i}\right)=C
$$

where $S$ is such that $x_{i}=\partial S / \partial y_{i}$, and $F \equiv F\left(x_{i}, y_{i}\right)=C$ is an integral of the canonical equations. Instead of putting $F=F_{0}$ and finding the corresponding value of $S$, he puts

$$
S=S_{0}+\mu S_{1}+\cdots, \quad F=F_{0}+\mu F_{1}+\cdots,
$$

and, expanding in powers of $\mu$, determines $S_{0}, S_{1}, \ldots$ successively. Various difficulties arise. In astronomical applications $S$ is not generally expansible in powers of $\mu$ but in powers of $\sqrt{ } \mu$, just as in the inversion of $y=a_{1} x+a_{2} x^{2}+\cdots$, when $a_{1}=0, x$ is expansible in powers of $\sqrt{ } \frac{x}{y}$. Again we usually require the solution to consist of periodic terms only, and special ways of defining the arbitrary constants must be used to effect this.

An equation frequently arising is

$$
\frac{d^{2} x}{d t^{2}}+x\left(1+\mu q_{1} \cos t+\mu^{2} q_{2} \cos 2 t+\cdots\right)=0 .
$$

This equation has been treated by many writers. $\dagger$

2. Integrals. - An integral of the equations

$$
\frac{d x_{i}}{d t}=X_{i} \quad(i=1,2, \cdots, n)
$$

* Les nouvelles méthodes de la mécanique céleste, chaps. IX, XI, etc.

+ For references see 'Tisserand, Méc. cél., vol. 3, chaps. I, II. 
is defined to be a function of the $x_{\text {s }}$ such that when it is differentiated and the values of the $d x_{i} / d t$ substituted, the result is identically zero. When we consider integrals which are one-valued analytic functions of the $x_{i}$, ten are known in the problem of three bodies; Bruns,* Poincare, $\dagger$ and Painlévé $f$ have considered the question of the existence of other integrals and the answer is always in the negative.

3. Integral Invariants. $\$$ Suppose that we have a $p$-tuple integral

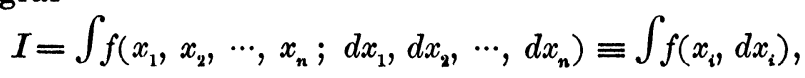

where $f$ is algebraic and homogeneous of order $p$ with respect to the $d x_{i}$, and the $x_{i}$ satisfy the equations $d x_{i} / d t=X_{i}$, the limits of integration being over a certain range of values of the $x_{i}$. Let the equations be integrated so that the $x_{i}$ are expressed in terms of $t$, the arbitraries being the values of the $x_{i}$ for $t=0$. Substituting, we obtain

$$
I=\int \psi\left(t, a_{i}, d a_{i}\right) .
$$

Take a range $R_{0}$ of possible values of the $a_{i}$ and integrate along this range for $t=0$; we obtain an integral

$$
I_{0}=\int_{R_{0}} \psi^{\prime}\left(0, a_{i}, d a_{i}\right) \text {. }
$$

To each value of $a_{i}$ in $R_{0}$ will correspond a set of values of $x_{i}$ at time $t$; thus to the range $R_{0}$ will correspond a range $R$ at time $t$ giving

$$
I=\int_{R} \psi\left(t, a_{i}, d a_{i}\right)
$$

If $I=I_{0}$ for all values of $t$, then $I$ is an integral invariant. An example is furnished by the motion of an incompressible fluid; the constant volume of any portion gives an integral invariant. The "circulation" in vortex motion furnishes another.

A relative invariant is one only existent for closed folds -using the language of $n$ dimensional geometry ; an absolute invariant is one existent for open folds. A relative invariant of order $p$ can be transformed into an absolute invariant of order $p+1$ when the function under the integral sign is also a polynomial in the $d x_{i}$, in a manner analogous to

* Acta Math., vol. 11.

† Loc. cit., chap. V.

¥ Comptes rendus, vol. 130, p. 1699.

$\S$ Poincaré, l. c., chap. XXII. 
Stokes's theorem for transforming from a line to a surface integral.

To an integral invariant of the equations $d x_{i} / d t=X_{i}$ corresponds an integral of the equations of variations (see section 7 below), and vice versa. As the equations of variations of the canonical equations of dynamics possess at least one integral, an integral invariant always exists for the equations of dynamics.*

One of the chief uses of integral invariants is as a means of verifying the results when the problem has been solved. If we confine ourselves to special forms of integral invariants, the maximum number possible in the three bodies problem can be stated and some of them are actually known. The unknown ones may not exist at all. Poincaré suggests that perhaps the method of Bruns, referred to above, might be used to furnish existence theorems.

$5 . \dagger$ Divergent Series. $\$-A s$ many of the series which are used in astronomy are divergent, it is necessary to consider how the approximate values of the functions which they are supposed to represent may be obtained from them.

Suppose a divergent series

$$
f_{0}+\mu f_{1}+\cdots
$$

to represent formally a function $\varphi$, and let $\varphi_{p}$ be the sum of the first $p+1$ terms of the series. If

$$
\lim _{\mu=0} \frac{\varphi-\varphi_{p}}{\mu^{p}}=0
$$

the series is said to represent $\varphi$ asymptotically and the first $p$ terms will give an approximation to $\varphi$, which will be the more exact the smaller $\mu$ is. The greatest value of $p$ is generally the number of the smallest term and $p$ is greater the smaller $\mu$ is.

A method analogous to Cauchy's proves that it is possible to use such series as solutions of the equations $d x_{i} / d t=X_{i}$, and it furnishes a limit to the error committed. The main point is that only a limited degree of approximation is possible ; the practical effect in astronomy is to limit the time during which the series are available.

* Following Poincaré, we consider the equations of dynamics to be such that when put into a canonical form, they have the integral $F=C$, where $F$ is independent of $t$ explicitly.

† Owing to lack of time no. 4 (Least action) of the syllabus distributed at the meeting was omitted.

† Poincaré, $l$. c., chaps. VIII, XIII ; Borel, Leçons sur les séries divergentes, chap. I. 
Another form of divergence arises with series like

$$
A_{0}+\sum_{m_{1}} \Sigma_{m_{2}} \frac{A_{m_{1}, m_{2}}}{m_{1} n_{1}+m_{2} n_{2}} \cos \left(m_{1} n_{1}+m_{2} n_{2}\right) t
$$

where $m_{1}, m_{2}$ are positive or negative integers, $A$ is a nonvanishing coefficient and $n_{1}, n_{2}$ are any numbers. For convergence it is necessary that

$$
\left|\frac{A}{m_{1} n_{1}+m_{2} n_{2}}\right|<\varepsilon, \text { a finite number, }
$$

which cannot hold if $n_{1} / n_{2}$ is commensurable with unity. In applications $n_{1}, n_{2}$ are observed numbers and therefore determined to a limited number of places of decimals. It may be shown that we can generally find numbers $n_{1}^{\prime}, n_{2}^{\prime}$ as close as we wish to $n_{1}, n_{2}$ such that the series either diverges or converges as we desire ; in the latter case the convergence is not uniform.

The final result for astronomical series is as follows :

The canonical equations

where

$$
\frac{d x_{i}}{d t}=\frac{\partial F}{\partial y_{i}}, \frac{d y_{i}}{d t}=-\frac{\partial F}{\partial x_{i}} \quad(i=1, \ldots, p),
$$

$$
F=F_{0}+\mu F_{1}+\mu^{2} F_{2}+\ldots \quad \text { (convergent), }
$$

are satisfied formally by

where

$$
\begin{aligned}
& x_{i}=x_{i}^{(0)}+\mu x_{i}^{(1)}+\ldots, \\
& y_{i}=w_{i}+\mu y_{i}^{(1)}+\ldots,
\end{aligned} \quad w_{i}=n_{i} t+\tilde{\omega}_{i}
$$

(3) $x_{i}^{(k)}\left(\right.$ or $\left.y_{i}^{(k)}\right)=A_{0}+\Sigma \frac{B \cos \left(m_{1} w_{1}+\ldots+m_{p} w_{p}+h\right)}{m_{1} n_{1}+\ldots+m_{p} n_{p}}$;

the $m_{j}$ are integers, $n_{i}, \widetilde{\omega}_{i}, h, A_{0}, B$ constants, and the sign of summation includes all terms the type of which is written down (the coefficient $B$ is different for each term). By what has just been said we may consider the series (3) as convergent, but the series (2) do not converge. If (2) converged, it is shown that another integral of (1) would exist-contrary to the theorems of section 2 above. This does not necessarily imply instability ; it may be only a defect of our analytical mode of representation.

6. Periodic Solutions.-A system is said to be in periodic motion when, after the lapse of a certain interval, its posi- 
tion and motions are the same as at the beginning of the interval. For $n$ bodies the definition is extended to their relative positions and motions. There is no general method of finding all the periodic solutions of a given system, but existence theorems will deduce a range of them from a known one. There may be separate systems of periodic solutions ; the method deals with only one system at a time.

Poincaré uses different kinds of periodic solutions which may be exhibited for the problem of three bodies as follows :

Let $y_{1}, y_{2}, y_{3}, y_{4}, y_{5}, y_{6}$ represent the six angles used in the motion of two small bodies about a third large one ; $y_{1}, y_{4}$, the angular positions at time $t ; k_{1}, k_{2}, k_{3}, k_{4}, k_{5}$ any positive or negative integers.

A periodic solution exists if $y_{1}-y_{6}, y_{2}-y_{6}, y_{3}-y_{6}, y_{4}-y_{6}$, $y_{6}-y_{6}$ increase respectively by $2 k_{1} \pi, 2 k_{2} \pi, 2 k_{3} \pi, 2 k_{4} \pi, 2 k_{6} \pi$ when $t$ increases by a period.

The classification is shown by the following tree :

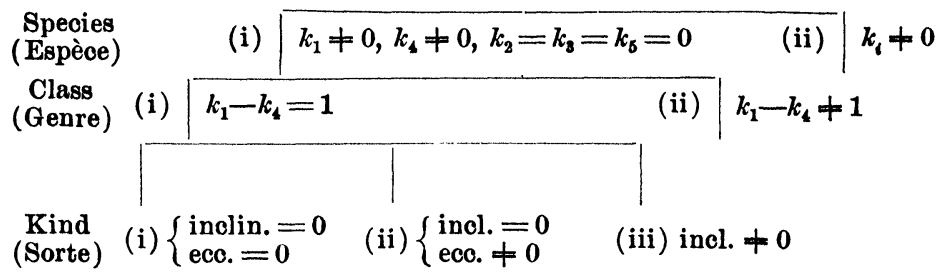

Periodic solutions of the first class* are those in which the relative orbits are closed curves with one turn round the primary body. These include the orbits of Hill $\dagger$ and Darwin.t

Poincaré's method is to suppose that a periodic solution exists for a particular value of $\mu$ and then to prove the existence for a range of values of $\mu$. There may be several periodic solutions for a given $\mu$. As $\mu$ is varied, they will appear and disappear in couples like the imaginary roots of an algebraic equation. 'The results differ according as, in the equations $d x_{i} / d t=X_{i}$, the $X_{i}$ do or do not contain $t$ explicitly, and according as the equations do or do not admit of an integral. The application to the orbits of Hill and Darwin is immediate, these being of the first kind; the second and third kinds, although they can exist, await applications.

* Poincaré, l. c., ohap. III.

+ Amer. Jour. Math., vol. 1.

$\ddagger$ Acta Math., vol. 21. See also S. S. Hough, ibid., vol. 24 ; and Schwarzschild, Astr. Nach., vol. 147. 
7. Characteristic Exponents.*-Suppose that the equations $d x_{i} / d t=X_{i}$ admit a known periodic solution

$$
x_{i}=\phi_{i}^{\prime}(t) \text {, }
$$

and that we try to obtain a solution (not necessarily periodic)

$$
x_{i}=\psi_{i}(t)+\xi_{i},
$$

not very different from the former. We substitute the latter in the differential equations and, omitting squares and products of the $\xi_{i}$, obtain the equations of variations

$$
\frac{d \xi_{i}}{d t}=\xi_{1} \frac{\partial X_{i}}{\partial x_{1}}+\xi_{2} \frac{\partial X_{i}}{\partial x_{2}}+\cdots+\xi_{n} \frac{\partial X_{i}}{\partial x_{n}} .
$$

The general solution of these is

$$
\xi_{i}=A_{1} e^{a_{1} t} S_{i 1}+\cdots+A_{n} e^{a_{n} t} S_{i n},
$$

where the $A_{i}$ are arbitraries, $S_{i j}$ known periodic functions of $t$, and the $\alpha_{i}$ constants which depend only on the constants present in the equations of variations.

The $\alpha_{i}$ are called the characteristic exponents. There is the usual modification of the solution when two or more of the $a_{i}$ are equal or when some of them are zero: terms are introduced with $t, t^{2}, \cdots$ as factors. These terms do not necessarily imply instability.

The discussion turns on (i) whether the $\alpha_{i}$ are real, imaginary, or complex ; (ii) whether one or more of them is zero ; (iii) whether two or more of them are equal. The three cases depend mainly on the presence of $t$ explicitly in the $X_{i}$, and on the existence of an integral of the original equations. The equations of dynamics (see the first footnote to p. 107) give two exponents zero and the others equal and opposite in pairs. In most problems of celestial mechanics the $\alpha$, are expanded in powers of $\sqrt{\mu}$.

8. Periodic solutions of the second class $†$ give more than one revolution round the primary before the curve is reëntering. The only method at present available is to investigate the existence of such solutions near a solution of the first class. Consider one solution of the equations of variations

$$
\xi_{i}=A e^{a t \sqrt{-1}} S
$$

* Poincaré, l. c., chap. IV.

† Poincaré, l. c., chaps. XXVIII., XXX I. 
and let $T$ be the period of the first-class solution and therefore of $S$, and suppose $a$ to be real. The $\xi_{i}$ are then expressible by sines and cosines of the angles at and of multiples of $2 \pi t / T$. If $\alpha=2 m / k T, m, k$ being integers, then after $k$ revolutions the $\xi_{i}$ take up again their old values and the period is $k T$. 'The difficulties to be dealt with arise from the fact that only the first powers of the $\xi_{i}$ are at first included. Special applications are made to the Darwin orbits, and a connection between the conditions for the existence of a second-class orbit and the passage from stability to instability of the Darwin orbits is shown.

9. Periodic Solutions of the Second Species.-Poincare* conceives a way in which these may exist. He supposes that two very small bodies are describing orbits round a large one and that the orbits are so placed that a collision very nearly takes place at definite intervals. The orbits are ellipses whose elements remain practically constant except near each collision point where they are suddenly and greatly changed. Thus all the elements may vary and the motion may be periodic. It may be possible by methods of continuity to deduce periodic solutions in which collisions will not nearly occur ; but such orbits would probably be very unstable.

10. Asymptotic Solutions. $\dagger$-Suppose that we have a problem of dynamics with two degrees of liberty. Using the periodic solution, and then a near solution, we obtain results of the form

$$
\begin{aligned}
& x_{i} \\
& y_{i}
\end{aligned}=\varphi_{i}(t)+\text { series in powers of } A e^{a t}, A^{\prime} e^{-a^{t}} \quad(i=1,2)
$$

where $\varphi_{i}(t)$ and the coefficients of the series are periodic. Suppose $a$ real and positive ; then $A, A^{\prime}$ are real. If $A=0$, the solution approximates to $\varphi_{i}(t)$ for $t$ very great, and the orbit, which starts with a slight modification of the periodic solution, soon differs much from it, but as time goes on approaches it more and more nearly. The solution is called asymptotic. A second kind of asymptotic solution is obtained with $A^{\prime}=0, A \neq 0$.

We may discuss the problem by considering $x_{1}, x_{2}, y_{1}$ as coördinates of a point in space; $y_{2}$ is determined from the energy equation $F\left(x_{1}, x_{2}, y_{1}, y_{2}\right)=C$, where $C$ is a given constant. Then if $t, A^{\prime} e^{-a t}$ be treated as parameters, the point $\left(x_{1}, x_{2}, y_{1}\right)$ always lies on a surface, called asymptotic. Simi-

* Loc. cit., chap. XXXII.

† Poincaré, l. c., chaps. VII, XXXIII. 
larly we have a second asymptotic surface for $A^{\prime}=0, A \neq 0$. The representation may be changed by transformation of coördinates if necessary for convenience of discussion. The periodic solution $\left(A=0, A^{\prime}=0\right)$ lies on both surfaces and therefore on their intersection. The particular case of the three bodies problem in which the third body is very small (of "zero" mass) and the other two describe circular orbits about one another can be treated. With properly chosen coördinates instead of $x_{1}, x_{2}, y_{1}$, it is shown that two curves on an asymptotic surface which correspond to trajectories never cut one another, and that two similar curves on different asymptotic surfaces may cut.

The doubly asymptotic solution is one which approaches a periodic solution when $t=+\infty$ and also when $t=-\infty$. The periodic solutions thus approached may be the same (homoclinic) or different (heteroclinic). A homoclinic orbit never cuts itself. There are two kinds of homoclinic solutions and an infinite number of each kind such that a trajectory never cuts one of its own kind but cuts one of the other kind an infinite number of times. Very little is known of heteroclinic solutions.

11. Stability. - In the problem of $n$ bodies the sufficient conditions for stability appear to be :

$1^{\circ}$. That the bodies never get infinitely distant from one another ;

$2^{\circ}$. That their mutual distances never descend below a certain limit;

$3^{\circ}$. That each body passes an infinite number of times as near as we wish to any point through which it has once passed ;

$4^{\circ}$. That a small external disturbance shall not affect the fulfilment of conditions $1^{\circ}, 2^{\circ}, 3^{\circ}$.

It is not known whether these are all necessary. In the general problem we do not know whether any one of them is satisfied. Poincaré states the first three conditions* and proceeds to investigate the third in some detail. He shows that when the bodies are started so as always to remain within a closed surface, those that satisfy $3^{\circ}$ are the rule and the others are the exception, the former being infinite in number compared with the latter. The proof depends on the existence of an integral invariant. In a particular case of the sun-rarth-moon system the numerical limits for $1^{\circ}, 2^{\circ}$ have been found. $\dagger$

* Loc. cit., chap. XXVI.

† Hans Haffel, Inaugural Dissertation, Göttingen, 1900. 


\section{Literature.}

Full references to the literature of celestial mechanics are given in the following works :

Gautier: "Essai historique sur le problème des trois corps." Paris, 1817.

Cayley : "On the progress of the solution of certain problems in dynamics." B. A. Reports, 1862.

Dziobek: Die mathematischen Theorien der PlanetenBewegungen. Leipzig, 1888.

Tisserand: Traité de mécanique céleste. Paris, vol. 1, 1889 ; vol. 2 , 1891 ; vol. 3 , 1894 ; vol. $4,1896$.

Brown : Introductory treatise on the lunar theory. Cambridge, 1896.

Whittaker: "Report on the progress of the solution of the problem of three bodies. B. A. Reports, 1899.

Wislicenus: Astronomischer Jahresbericht. Berlin, vol. 1 for 1899 , vol. 2 for 1900 .

It may be added that most of Gylden's work is to be found in the volumes of the Acta Mathematica and in the "Traite analytique des orbites absolues des huit planètes principales" (Stockholm, vol. 1, 1893).

The treatise of Poincaré, frequently referred to above, has been mainly used in the preparation of these lectures. It consists of three volumes published in $1892,1893,1899$, respectively, and is an expansion of the memoir in vol. 13 (1890) of the Acta Mathematica entitled, "Sur le problème des trois corps et les équations de la dynamique." Most of this author's other papers on these subjects will be found in the volumes of the Bulletin Astronomique.

\section{THE HAMBURG MEETING OF THE DEUTSCHE MATHEMATIKER-VEREINIGUNG, SEPTEMBER, 1901.}

The annual meeting of the Deutsche Mathematiker-Vereinigung was held at Hamburg, September 22-28. In the absence of the president of the society, Professor von Dyck, Professor Hilbert occupied the chair. At the business meeting Professor Gutzmer reported on a plan for changing the Jahresbericht into a monthly periodical to be the official organ of the society, and to contain mathematical papers, univer- 